\title{
Molecularly determined total tumour load in lymph nodes of stage I-II colon cancer patients correlates with high-risk factors. A multicentre prospective study
}

\author{
Iban Aldecoa ${ }^{1}$ - Begoña Atares ${ }^{2}$ - Jordi Tarragona ${ }^{3}$ - Laia Bernet ${ }^{4}$. \\ Jose Domingo Sardon ${ }^{5}$ - Teresa Pereda ${ }^{6}$ - Carlos Villar ${ }^{7}$ - M. Carmen Mendez ${ }^{8}$. \\ Elvira Gonzalez-Obeso ${ }^{8}$ - Kepa Elorriaga ${ }^{9}$ - Guadalupe Lopez Alonso ${ }^{10}$. \\ Javier Zamora ${ }^{11}$ • Nuria Planell ${ }^{12}$ • Jose Palacios ${ }^{13}$ • Antoni Castells ${ }^{14}$. \\ Xavier Matias-Guiu ${ }^{3} \cdot$ Miriam Cuatrecasas $^{1,15}$
}

Received: 6 April 2016 / Revised: 17 June 2016 / Accepted: 7 July 2016 / Published online: 22 July 2016

(C) The Author(s) 2016. This article is published with open access at Springerlink.com

\begin{abstract}
Stage I-II (pN0) colorectal cancer patients are surgically treated although up to $25 \%$ will eventually die from disease recurrence. Lymph node (LN) status is an independent prognostic factor in colorectal cancer (CRC), and molecular tumour detection in LN of early-stage CRC patients is associated with an increased risk of disease recurrence and poor survival. This prospective multicentre study aimed to determine the relationship between LN molecular tumour burden and conventional high-risk factors in stage I-II colon cancer patients. A total of $1940 \mathrm{LN}$ from 149 pathologically assessed pN0 colon cancer patients were analysed for the amount of tumour cytokeratin 19 (CK19) messenger RNA (mRNA) with
\end{abstract}

Previous presentations This work presented in part as an oral platform at the USCAP (United States and Canadian Association of Pathologists) 103rd Annual Meeting, San Diego, CA. March 1-7, 2014.

Electronic supplementary material The online version of this article (doi:10.1007/s00428-016-1990-1) contains supplementary material, which is available to authorized users.

Miriam Cuatrecasas

mcuatrec@clinic.ub.es

1 Pathology Department, Centre de Diagnòstic Biomèdic (CDB), Hospital Clínic, University of Barcelona, Escala 3, Planta 5.

Villarroel 170, Barcelona 08036, Spain

2 Pathology Department, Alava University Hospital, Vitoria-Gasteiz, Spain

3 Pathology Department, Hospital Arnau de Vilanova, Lleida, Spain

4 Pathology Department, Hospital L. Alcanyis, Xativa, Spain

5 Surgery Department, Alava University Hospital, Txagorritxu, Spain

6 Pathology Department, Hospital Costa del Sol, Marbella, Spain

7 Pathology Department, Hospital Reina Sofia, Cordoba, Spain the quantitative reverse transcription loop-mediated isothermal amplification molecular assay One-Step Nucleic Acid Amplification. Patient's total tumour load (TTL) resulted from the sum of all CK19 mRNA tumour copies/ $\mu \mathrm{L}$ of each positive LN from the colectomy specimen. A median of $15 \mathrm{LN}$ were procured per case (IQR 12;20). Molecular positivity correlated with high-grade $(p<0.01)$, mucinous/signet ring type $(p=0.017)$, male gender $(p=0.02)$, number of collected LN $(p=0.012)$ and total LN weight per case $(p<0.01)$. The TTL was related to $\mathrm{pT}$ stage $(p=0.01)$ and tumour size $(p<0.01)$ in low-grade tumours. Multivariate logistic regression showed independent correlation of molecular positivity with gender,

8 Pathology Department, Hospital Severo Ochoa, Leganes, Madrid, Spain

9 Pathology Department, Hospital Onkologikoa, San Sebastian, Spain

10 Pathology Department, Hospital 12 Octubre, Madrid, Spain

11 Biostatistic Unit, Hospital Ramon y Cajal, Madrid, Spain

12 Gastroenterology Department and Bioinformatics Unit, CIBERehd, IDIBAPS, Hospital Clinic, University of Barcelona, Barcelona, Spain

13 Pathology Department, Hospital Ramon y Cajal, Madrid, Spain

14 Gastroenterology Department, Hospital Clinic, University of Barcelona, IDIBAPS, CIBERehd, Barcelona, Spain

15 CIBERehd, and Banc de Tumors-Biobanc Clinic-IDIBAPS-XBTC, Hospital Clinic, Barcelona, Spain 
tumour grade and number of fresh LN [AUC $=0.71(95 \%$ $\mathrm{CI}=0.62-0.79)]$. Our results show that lymph node CK19 mRNA detection correlates with classical high-risk factors in stage I-II colon cancer patients. Total tumour load is a quantitative and objective measure that may help to better stage early colon cancer patients.

Keywords Colorectal neoplasms $\cdot$ Neoplasm staging · Molecular pathology $\cdot$ Lymph nodes $\cdot$ Cytokeratin 19

\section{Introduction}

Surgical resection with no adjuvant therapy is recommended for most stage I-II colorectal cancer (CRC) patients, except for selected high-risk stage II patients given the significant impact of chemotherapy on stage III disease [1,2]. Although there is evidence that pathological nodal staging is far from being optimal, current NCCN guidelines are based on haematoxylin and eosin (HE) lymph node (LN) staging, [3-7]. Its major weakness is the limited scope of histological $\mathrm{LN}$ analysis, based on a small sample provided by $2-5 \mu \mathrm{m} \mathrm{LN}$ sections, which comprise less than $0.5 \%$ of the entire $\mathrm{LN}$, and it may lead to false negative diagnoses [3, 7-9]. This may partly explain why up to $25 \%$ of CRC patients with histologically negative $\mathrm{LN}$ die from recurrent disease after a potentially curative surgical resection. Some of these patients may have had undetected LN metastases [3, 10, 11].

The use of additional techniques, i.e. immunohistochemistry (IHC) or reverse transcriptase polymerase chain reaction, makes it possible to find LN tumour burden not detected with conventional $\mathrm{HE}$ analysis in 25 to $50 \%$ of CRC patients, due to both increased sensitivity and the more extensive study than usually permitted by histological sections [3, 6, 7, 10-17].

Although the prognostic value of LN molecular tumour detection in early-stage CRC is controversial [14-18], there are enough data to support the use of more sensitive (i.e. molecular) methods of LN staging. As stated in three metaanalyses, the molecular detection of tumour cells in regional $\mathrm{LN}$ of stage I-II CRC patients is associated with an increased risk of disease recurrence and poor survival $[3,10,11]$.

Most studies have focused in dichotomic (positivenegative) or semi-quantitative scales (isolated tumour cells (ITC), micro- and macrometastases) to assess molecular results $[8,10,11,14,15,17,19,20]$. The molecular assay OneStep Nucleic Acid Amplification (OSNA; Sysmex Corporation, Kobe, Japan) is a quantitative method which analyses the entire LN. It amplifies cytokeratin 19 (CK19) mRNA from LN tissue lysates using the reverse transcription loop-mediated isothermal amplification (RT-LAMP) method [21]. CK19 mRNA was selected among other CRC markers showing the highest diagnostic performance and reproducibility, with $94.9-95.2 \%$ sensitivity and $97.7-97.9 \%$ specificity
[22]. The system has been validated for breast and colon cancer LN assessment, providing results comparable to extensive histological and IHC LN analysis [8, 9, 21, 23-28]. The amount of CK19 mRNA detected correlates with the size of the metastatic foci $[8,9,21,27,29]$. It also makes it possible to calculate the total tumour load (TTL) present in a given patient, by adding all CK19 mRNA copies from each positive LN of a colectomy specimen [27, 28].

In this multicentre prospective study, we tried to correlate the TTL, as determined by OSNA, with classical clinical and pathologic high-risk factors, in an effort to determine whether the TTL could be used as an additional factor to better select stage I-II patients at risk of recurrence. Such an approach is now widely used in the treatment of breast cancer [27, 30, 31].

\section{Materials and methods}

\section{Study sample}

This is a prospective observational study including 10 institutions. Inclusion criteria were patients over 18 years old, with primary histologically confirmed colon cancer, $\mathrm{cN} 0$ preoperative diagnosis and positive CK19 IHC of the primary tumour. Exclusion criteria included rectal tumours, non-invasive $\mathrm{pTis}$ and pT0 tumours, positive LN on HE, synchronous tumours or other malignancies, $\mathrm{cN} 1$, gross adipose tissue involvement by the tumour, metastatic cancer, neo-adjuvant chemotherapy, familial adenomatous polyposis, carcinomas on inflammatory bowel disease and presence of stent-type intraluminal devices.

\section{Study procedure}

\section{Sample processing and fresh lymph node procuring}

Fresh $\mathrm{LN}$ procurement from the mesocolon fat was performed within 50 min after surgical excision. When immediate LN dissection was not possible, the surgical specimen was kept up to $3 \mathrm{~h}$ in the refrigerator at $4{ }^{\circ} \mathrm{C}$ until $\mathrm{LN}$ dissection was done. During the LN harvesting process, the dissection area was kept cold by putting a thick layer of chopped ice under an elevated metallic surface and covered with a clean filter paper for LN dissection (Figure available at Online Resource 1a). Microcentrifuge tubes were also kept cold by punching them in chopped ice (Figure 2 available at Online Resource 1b). We first detached the mesocolon fat from the colon wall with a surgical blade. pT4 tumours corresponded to antimesenteric serous tumour infiltration, and pT3 tumours corresponded only to specimens with minimal tumour infiltration of the mesocolon. We then dissected one by one all LN from the mesocolon fat using different clean areas of the surgical blade for small $\mathrm{LN}$, or changing it after each $\mathrm{LN}$ dissection. When a LN was grossly suspicious of being positive, a cytology 
touch-prep was performed to confirm or discard metastasis. Positive cases were discarded. All freshly dissected LN were analysed by both methods, HE and OSNA, using a modified protocol from previous studies $[8,9,21]$. Each $\mathrm{LN}$ was numbered and cut along the long axis. A central 1-mm slice was submitted for conventional formalin-fixation paraffin-embedding (FFPE) and HE analysis. The rest of the LN was stored at $-80^{\circ} \mathrm{C}$ in microcentrifuge tubes for 1 to 7 days until deferred OSNA analysis was performed. Lymph nodes with weight $\leq 0.07 \mathrm{~g}$ (average $5.5 \mathrm{~mm}$ ) were defined as small.

After fresh LN harvesting, the specimen was fixed overnight in $10 \%$ neutral buffered formalin. Then, the mesocolon fat was re-examined for remaining LNs, which were submitted only for conventional histopathology analysis.

\section{CK19 immunohistochemistry}

CK19 IHC was performed on representative sections of all the primary colon carcinomas to ensure reliable negative molecular CK19 mRNA results. A 2- $\mu$ m section of each primary tumour was mounted on FLEX IHC microscope slides and pre-treated in PT-LINK (Dako, Glostrup, Denmark). Incubation for $20 \mathrm{~min}$ with the primary CK19 antibody (CK19 mouse monoclonal, clone RCK108; IR615 pre-diluted. Dako) was performed in the AutostainerLink 48 (Dako). Membranous staining with or without cytoplasm staining of $\geq 10 \%$ of the tumour cells was defined as positive IHC in colon carcinomas (Fig. 1a, b).

\section{Pathology report and LN staging}

LN staging and pathology report were performed from the analysis of HE stains according to the AJCC/UICC TNM, 7 th edition $[32,33]$. Tumours $\geq 4 \mathrm{~cm}$ were defined as large. Pathologists and clinicians were both blinded to the OSNA results.

\section{OSNA procedure}

The OSNA method was performed at each institution following the manufacturer's instructions, using the protocol described by Tsujimoto et al. [21]. Briefly, LNs were weighed, homogenized with the lysis buffer Lynorhag (Sysmex) for mRNA stabilization and genomic DNA precipitation. After centrifugation for $1 \mathrm{~min}$ at $10,000 \times g$ at room temperature; a $2-\mu \mathrm{L}$ sample of the intermediate phase was mixed with the reagent LynoampBC (Sysmex). Analysis was performed using the RT-LAMP isothermal amplification method with the RD-100i automated gene-amplification system (Sysmex). The amount of CK19 mRNA amplified was detected by a change in turbidity upon precipitation of magnesium pyrophosphate (Fig. 1c). The result was correlated to CK19 mRNA copy number/ $\mu \mathrm{L}$ of the original lysate through calibrated standard curves containing different CK19 mRNA concentrations. In every assay, a standard positive control sample containing $5 \times 10^{3}$ copies $/ \mu \mathrm{L}$ of CK19 mRNA, and a negative control sample without CK19 mRNA were used for validation. The results were based on the number of CK19 mRNA copies $/ \mu \mathrm{L}$ obtained for each $\mathrm{LN}$. The cutoff value was 250 copies $/ \mu \mathrm{L}$, based on a previous study [9].

\section{Statistical analysis}

The Fisher exact test and Pearson's correlation coefficient were used for testing the association between categorical or numerical variables, respectively. The Mann-WhitneyWilcoxon and Kruskal-Wallis test were applied to compare groups' distributions. A $p<0.05$ was considered statistically significant.

Logistic regression analysis was used to predict the OSNA outcome. The backward stepwise algorithm was used to determine the best-fitting model. The classification avidity of the model was assessed by the ROC curve and a 10-fold crossvalidation technique was applied for the model validation. The Cohen's kappa was used to assess the degree of agreement between OSNA outcome and model prediction. All analyses were performed using $R$ statistical environment (V.3.1.0) [34].

\section{Results}

\section{Sample size and characteristics}

We analysed $3512 \mathrm{LN}$ from 211 colon cancer patients recruited in 10 hospitals between June 2012 and December 2013. We excluded 27 non-invasive tumours (9 pT0 and 18 pTis), 34 cases with HE-positive LN, and one patient with synchronous tumours. Finally, 149 stage I-II colon cancer patients met the study selection criteria. All primary tumours showed positivity for CK19 IHC. Demographic, clinical, pathological, and lymph node characteristics of the study sample and correlation with CK19 mRNA results are summarized in Table 1 and the study flow diagram (Fig. 2).

\section{Lymph node features and CK19 mRNA results}

Among the $2483 \mathrm{LN}$ procured from 149 cases, slightly over $78 \%$ of them were freshly isolated and submitted for OSNA analysis (1940 fresh LN vs 543 FFPE LN). Hence, we obtained a median of $15 \mathrm{LN}$ per case, of which $12 \mathrm{LN}$ were analysed by OSNA and HE.

CK19 mRNA positivity was observed in 76/149 patients (51\%). Most of those positive cases; i.e. $80 \%$, had only 1 to 3 positive LN. Thus, among all OSNA LN analysed, 9,8\% (190/1940) were positive. Association analysis showed that OSNA-positive cases harboured more freshly procured LN 


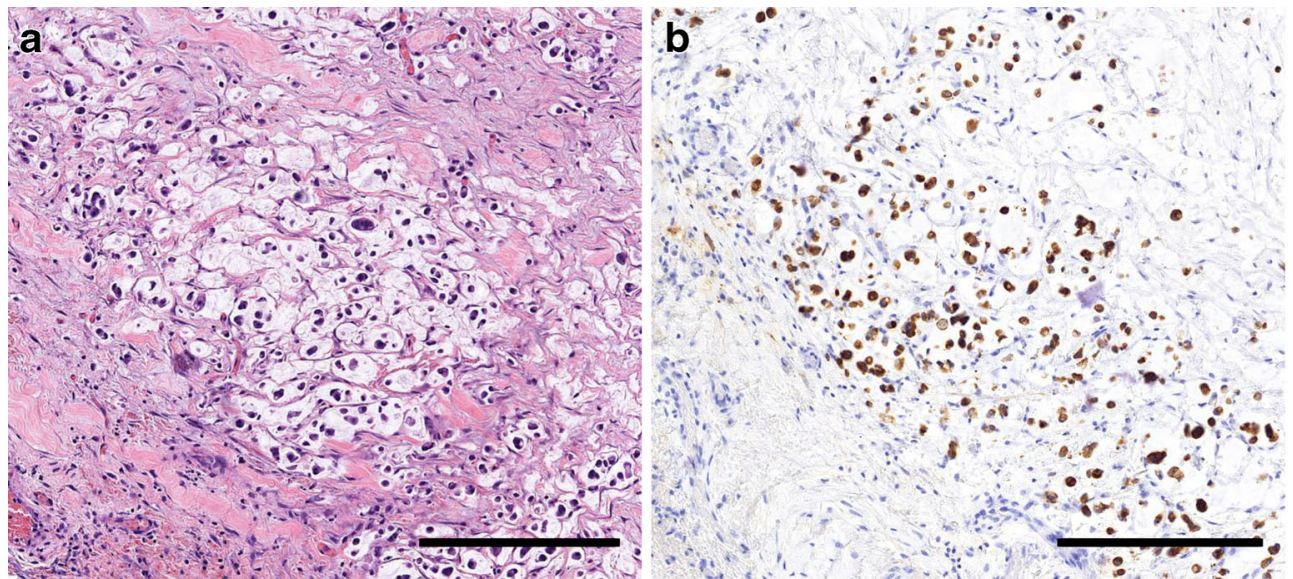

Fig. 1 a A primary high-grade mucinous carcinoma with signet ring cells. b Positive CK19 immunohistochemistry from the same case. c Amplification curve of $C K 19$ mRNA from a positive LN, assessed through turbidity variation ( $Y$-axis) over time ( $X$-axis, minutes). (Fig. $1 \mathrm{a}$, b, scale bar $250 \mu \mathrm{m})$

$(p=0.012)$ and had a higher total LN weight per case $(p<0.01)$ than negative ones (Fig. 3). Regarding the size of the freshly dissected LN, CK19 mRNA-negative LN were significantly smaller (i.e. weight $\leq 0.07 \mathrm{~g}$ ) than positive ones (29.3 vs $15.6 \%, p<0.01)$.

In conclusion, fresh $\mathrm{LN}$ dissection did not impede an effective $\mathrm{LN}$ dissection and was an efficient way to obtain the minimum number of $\mathrm{LN}$ required for $\mathrm{pN}$ assessment. Although a substantial part of cases harboured tumour burden, only a few LN were positive among them.

\section{High-risk factors and CK19 mRNA detection}

The pathological high-risk features of CRC that were related to the presence of tumour CK19 mRNA in the LN were as follows: mucinous/signet ring histological types, high histological grade and tumour size. Among demographic variables, male gender was significantly related to OSNA positivity. No relationship was found with $\mathrm{pT}$ stage, vascular invasion, macroscopic tumour configuration, or other features.

High-grade tumours were significantly larger than their low-grade counterparts $(p<0.01)$, with a trend between histological type and tumour size $(p=0.11)$. The logistic regression model including OSNA results as dependent variable and tumour size as independent variable adjusted by histological type or grade showed a no significant relation between tumour size and OSNA response ( $p=0.26$ and $p=0.15$, respectively).

The multivariate logistic regression analysis gave a reduced predictive model for OSNA molecular output including the independent variables gender, grade, and the number of fresh lymph nodes (Table 2). The receiver operating characteristic (ROC) curve was performed to test the avidity of the generated model to predict CK19 mRNA outcome, giving an $\mathrm{AUC}=0.71(95 \% \mathrm{CI}=0.62-0.79)$. With a decision boundary of 0.5 , the sensitivity, specificity, and accuracy were 61,68 , and $64 \%$, respectively. A 10-fold cross-validation was done for the model assessment, the resulting AUC was 0.67 (95\% $\mathrm{CI}=0.59-0.76)$ (Fig. 4). Therefore, male gender, high histological grade and higher yields of LN were independent predictors of CK19 mRNA positivity.

\section{Total tumour load}

The median TTL of CK19 mRNA copies/ $\mu \mathrm{L}$ among positive cases was 2015 (IQR: 940-6222.5). Cumulative TTL showed that $29 \%$ of patients $(22 / 76)$ had more than 5000 CK19 mRNA copies/ $\mu$ L. Although not significant $(p=0.18)$, there was a positive trend between CK19 mRNA copies $/ \mu \mathrm{L}$ and $\mathrm{pT}$ stage; while TTL in $\mathrm{pT} 1$ cases was 1280 CK19 mRNA copies/ $\mu \mathrm{L}$ and 1790 CK 19 mRNA copies $/ \mu \mathrm{L}$ in pT2, it rose to $3080 \mathrm{CK} 19 \mathrm{mRNA}$ copies $/ \mu \mathrm{L}$ in pT3 tumours (Fig. 3e). Surprisingly, the median TTL among pT4a tumours was 540 CK19 mRNA copies $/ \mu \mathrm{L}$. This could be explained by the inclusion of antimesenteric pT4 tumours rather than bulky tumours with marked adipose tissue infiltration, to the scarce amount of positive cases (5 among pT4a tumours), and by the fact than in 3 of them less than $65 \%$ of the LNs were analysed by OSNA. Distribution of the cases regarding their TTLs and divided by pT stage is provided as Supplementary Table 1 in Online Resource 2. Albeit also not significant, all OSNA positive cases with vascular invasion had $>2000 \mathrm{CK} 19$ mRNA copies $/ \mu \mathrm{L}$.

In order to evaluate the distribution of the TTL, it was categorized by quartiles and compared with classical high-risk factors (data not shown). When stratified by tumour grade, it showed that among low-grade tumours, TTL increased with tumour stage $(\mathrm{pT})(p=0.01)$ and tumour size $(p<0.01$; rho $=0.27)$ (Table 3$)$. This trend was not observed in high-grade tumours. Taken together, 
Table 1 Patient's demographics and specimen characteristics and correlation with CK19 mRNA results

\begin{tabular}{|c|c|c|c|c|}
\hline Clinical parameter & $n(\%)$ & $\begin{array}{l}\text { CK19 mRNA } \\
\text { negative } \\
\mathrm{n}(\%)\end{array}$ & $\begin{array}{l}\text { CK19 mRNA } \\
\text { positive } \\
\mathrm{n}(\%)\end{array}$ & $p$ value \\
\hline Cases & $149(100)$ & $73(49.0)$ & $76(51.0)$ & - \\
\hline Gender & & & & 0.02 \\
\hline Male & $97(65.1)$ & $41(42.3)$ & $56(57.7)$ & \\
\hline Female & $52(34.9)$ & $32(61.5)$ & $20(38.5)$ & \\
\hline Age (years)-median (IQR) & $67(61-75)$ & $68(61-74)$ & $66(61-75)$ & 0.89 \\
\hline \multicolumn{5}{|l|}{ Surgical specimen characteristics } \\
\hline Specimen size $(\mathrm{cm})$-median (IQR) & $20(15-25)$ & $20(15-25)$ & $19.5(15-25)$ & 0.81 \\
\hline Tumour size $(\mathrm{cm})^{\mathrm{a}} —$ median $(\mathrm{IQR})$ & $3(2-5)$ & $3(2-4)$ & $4(2-5.5)$ & 0.045 \\
\hline Large tumours $(>4 \mathrm{~cm})$ & $39(26.7)$ & $14(38.9)$ & $25(64.1)$ & 0.09 \\
\hline Small tumours $(\leq 4 \mathrm{~cm})$ & $107(73.3)$ & $57(53.3)$ & $50(46.7)$ & \\
\hline Tumour location & & & & 0.33 \\
\hline Right colon and caecum (incl. hepatic flexure) & $67(45)$ & $37(55.2)$ & $30(44.8)$ & \\
\hline Transverse colon & $14(9.4)$ & $5(35.7)$ & $9(64.3)$ & \\
\hline Left colon and sigmoid colon (incl. splenic flexure) & $68(45.6)$ & $31(45.6)$ & $37(54.4)$ & \\
\hline Macroscopic tumour configuration & & & & 0.57 \\
\hline Annular & $8(5.4)$ & $5(62.5)$ & $3(37.5)$ & \\
\hline Ulcerated & $65(43.6)$ & $33(50.8)$ & $32(49.2)$ & \\
\hline Polypoid & $75(50.3)$ & $34(45.3)$ & $41(54.7)$ & \\
\hline Other & $1(0.7)$ & $1(100)$ & $0(0)$ & \\
\hline Vascular invasion & & & & 0.45 \\
\hline No & $137(91.9)$ & $65(47.4)$ & $72(52.6)$ & \\
\hline Yes & $12(8.1)$ & $8(66.7)$ & $4(33.3)$ & \\
\hline Histological type & & & & 0.017 \\
\hline Adenocarcinoma & $136(91.3)$ & $71(52.2)$ & $65(47.8)$ & \\
\hline Mucinous/signet ring cell AC & $13(8.7)$ & $2(15.4)$ & $11(84.6)$ & \\
\hline Grade $^{b}$ & & & & $<0.01$ \\
\hline High grade & $22(15.0)$ & $4(18.2)$ & $18(81.8)$ & \\
\hline Low grade & $125(85.0)$ & $69(55.2)$ & $56(44.8)$ & \\
\hline pT & & & & 0.19 \\
\hline pT1 & $40(26.8)$ & $17(42.5)$ & $23(57.5)$ & \\
\hline pT2 & $31(20.8)$ & $20(64.5)$ & $11(35.5)$ & \\
\hline pT3 & $66(44.3)$ & $29(43.9)$ & $37(56.1)$ & \\
\hline pT4a & $12(8.1)$ & $7(58.3)$ & $5(41.7)$ & \\
\hline
\end{tabular}

$I Q R$ interquartile range, $A C$ adenocarcinoma

${ }^{a}$ In three patients, tumour size could not be assessed

${ }^{\mathrm{b}}$ In two patients, tumour grade could not be assessed

our results show that most patients had low TTLs. These values increased with tumour stage and followed different distributions among low- and high-grade tumours.

\section{Patient's follow-up}

At the time of writing of this manuscript, all patients had a follow-up of 2 years (median 33 months, IQR 25-32.5); $8.7 \%(13 / 149)$ patients recurred ( 3 died and 10 were alive with metastatic disease). Four of the cases were OSNA positive, with a median TTL of 4375 CK19 mRNA copies/ $\mu \mathrm{L}$ (range $360-47,600$ ); 2 of the 3 dead patients were OSNA positive.

\section{Discussion}

This study quantifies the amount of total tumour load within the lymph nodes of stage I-II colon cancer patients, and suggests that LN molecular detection of CK19 mRNA could 


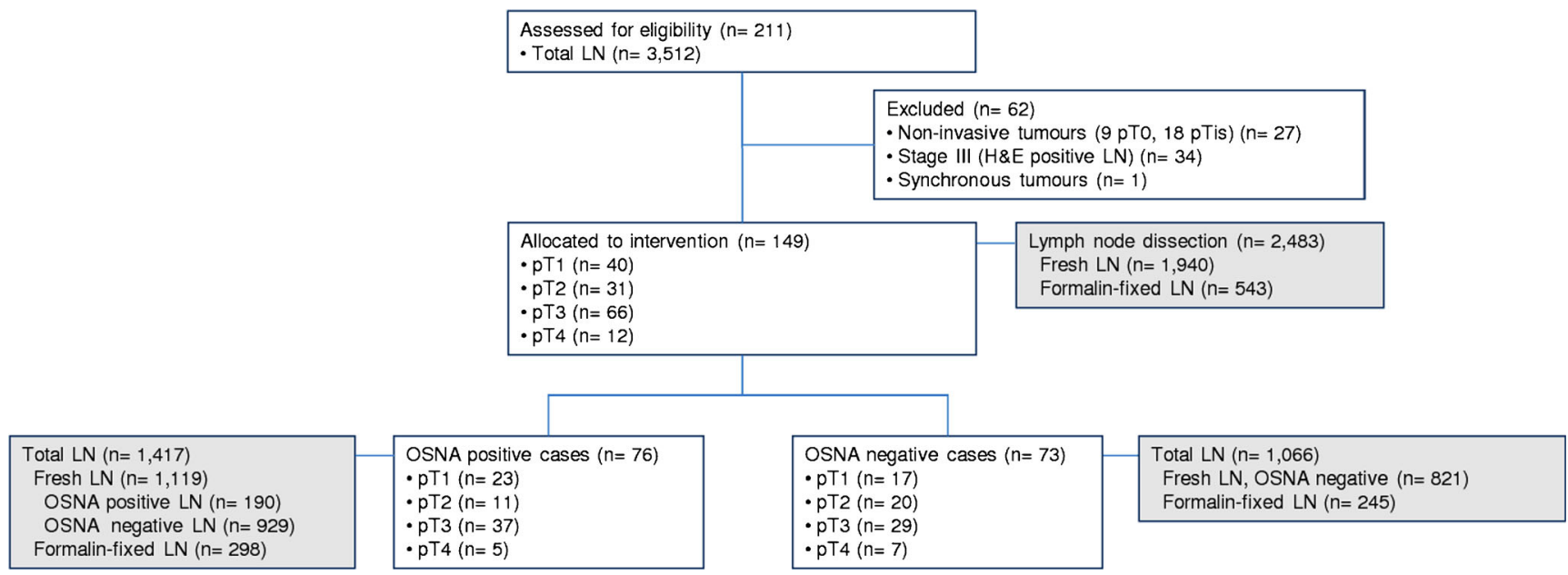

Fig. 2 Study flow diagram

become an objective indicator of risk in such individuals [11, 20, 28, 35]. Stage I-II CRC is amenable to complete surgical resection, but patients at risk of tumour recurrence are clinically and pathologically difficult to identify [36, 37]. In addition, cancer dissemination to LN may occur at early stages of tumour development $[6,38]$, potentially implying a poor
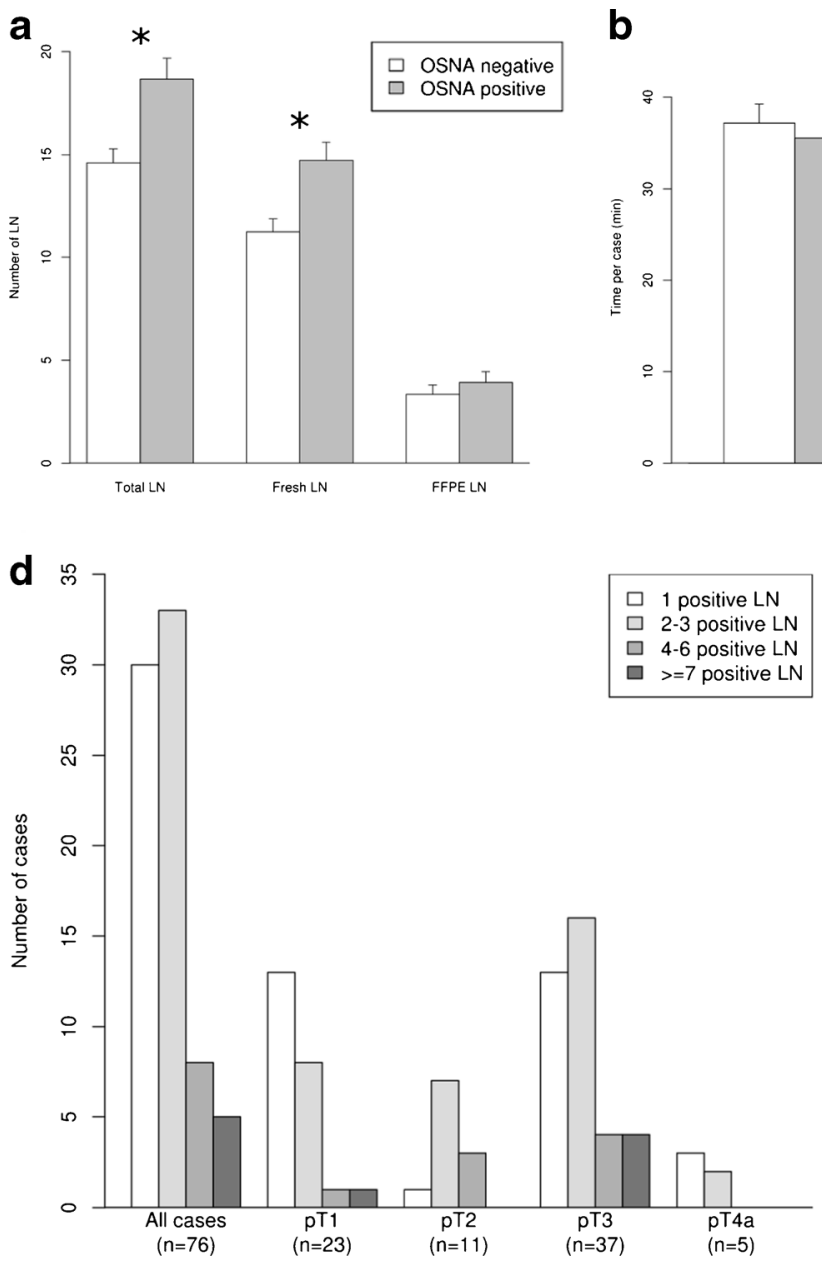

Fig. 3 OSNA results and correlation with $\mathrm{LN}$ retrieval and pT stage, regarding number of $\mathrm{LN}$ retrieved (a), time spent on fresh $\mathrm{LN}$ search in minutes (b) and total weight of fresh LN per case (gr) (c). OSNA positive cases held significantly more LN due to a higher fresh LN yields. d b
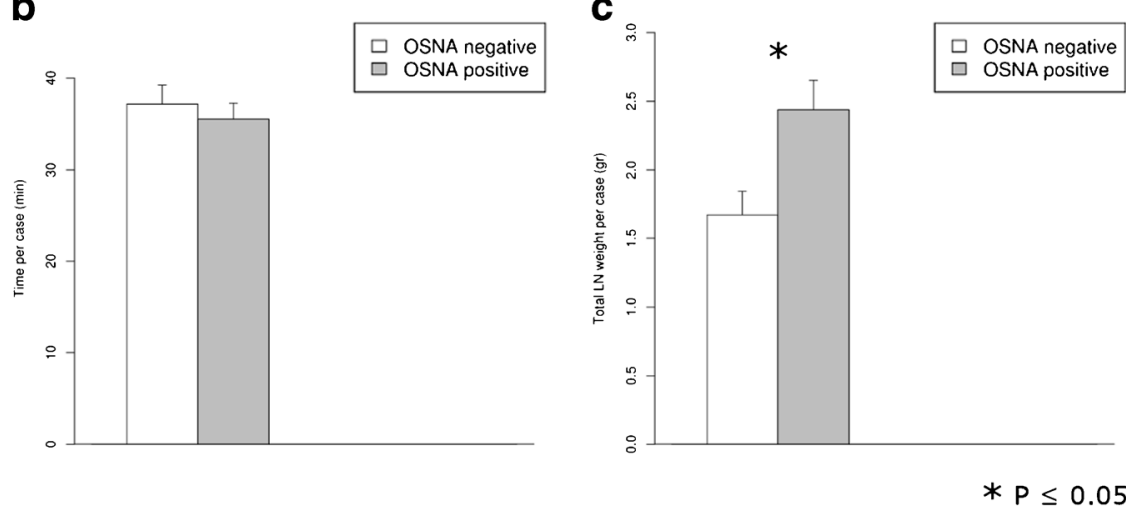

Shows that most cases in every pT stage held up to 3 OSNA positive LN. e Although not significant, a trend was observed between pT stage and TTL 
Table 2 Logistic regression of clinical and histological variables related to CK19 mRNA positivity

\begin{tabular}{|c|c|c|c|c|}
\hline \multirow[t]{2}{*}{ Variables } & \multicolumn{2}{|l|}{ Univariate model } & \multicolumn{2}{|c|}{ Multivariate model } \\
\hline & OR $(95 \% \mathrm{CI})$ & Wald test $p$ & OR $(95 \% \mathrm{CI})$ & Wald test $p$ \\
\hline Gender (male vs female) & $2.2(1.1-4.4)$ & 0.026 & $3.1(1.4-7.0)$ & 0.006 \\
\hline Age (years) & $0.99(0.96-1.03)$ & 0.74 & - & - \\
\hline Tumour size & $1.2(1.0-1.4)$ & 0.06 & - & - \\
\hline Tumour location & & 0.31 & - & - \\
\hline Transverse colon vs right colon and caecum (including hepatic flexure) & $2.2(0.7-7.9)$ & & & \\
\hline $\begin{array}{l}\text { Left colon and sigmoid colon (including splenic flexure) vs Right colon } \\
\text { and caecum (including hepatic flexure) }\end{array}$ & $1.4(0.7-2.9)$ & & & \\
\hline Macroscopic tumour configuration & & 0.59 & - & - \\
\hline Ulcerated vs annular & $1.6(0.4-8.4)$ & & & \\
\hline Polypoid vs annular & $2.0(0.5-10.4)$ & & & \\
\hline \multirow[t]{2}{*}{ Histological type (mucinous / signet ring cell AC vs adenocarcinoma) } & & 0.02 & - & - \\
\hline & $6.0(1.5-39.8)$ & & & \\
\hline Vascular invasion (yes vs no) & $0.5(0.1-1.5)$ & 0.21 & - & - \\
\hline Grade (high vs low) & $5.4(1.9-19.5)$ & $<0.01$ & $4.8(1.5-18.9)$ & 0.013 \\
\hline pT & & 0.20 & - & - \\
\hline pT2 vs pT1 & $0.4(0.2-1.1)$ & & & \\
\hline pT3 vs pT1 & $0.9(0.4-2.1)$ & & & \\
\hline pT4a vs pT1 & $0.5(0.1-1.9)$ & & & \\
\hline Fresh LN per case weight (gr) & $1.1(1.0-1.1)$ & $<0.01$ & $1.1(1.0-1.2)$ & 0.017 \\
\hline
\end{tabular}

$A C$ adenocarcinoma, gr grammes

prognostic impact $[3,7,11,15]$. Thus, the assessment of the nodal staging arises as a key factor for CRC therapeutic management $[3,10,11]$.

Our findings confirm the presence of undetected nodal tumour burden in early-stage colon cancer using the OSNA method. Compared to previous studies, we have the highest percentage of cases with CK19 mRNA detection in LN (i.e. $51 \%$ ) [23-25, 28]. Discrepancies may be due to differences in the LN collection and analysis, such as the fact that we analysed a larger amount of LN, including all evaluable LN by OSNA regardless of its size. Current guidelines recommend evaluating at least $12 \mathrm{LN}$ to achieve a reliable histological staging [16, 32, 33, 39-42]. In compliance with them, we obtained a median of $15 \mathrm{LN}$ per patient, of which 12 were freshly obtained and analysed by OSNA.

CRC lymph nodes are usually small, especially in early stages of the disease, but may still contain tumour burden, as size is not a good preoperative indicator of LN staging, or a predictor of the presence of tumour [43-45]. Although we identified a significant association between CK19 mRNA detection and larger LN size, we found CK19 mRNA in $15.6 \%$ of small LN. Furthermore, the multivariate analysis of OSNA results showed that the number of collected LN, the gender and the histologic grade were independent predictors of OSNA results. Our data highlights the importance of procuring any identifiable LN irrespective of its size [40-42, 45-47].
Our results show that the presence of CK19 mRNA in regional LN was associated with other classical high-risk factors such as mucinous/signet ring types, histologic high-grade, tumour size and male gender. Moreover, it is noteworthy the trend observed between TTL and pT stage, as pT3 had the highest TTL (3080 CK19 mRNA copies $\mu \mathrm{L}$ ) and rate of CK19 mRNA detection (37/66).

We also observed a different behaviour between low and high-grade tumours, with a significant orderly increase of TTL in regional LN with the increase of pT stage and tumour size in low-grade tumours. This phenomenon may be accounted for by the fact that low-grade tumours tend to follow an orderly sequence of accumulation of molecular alterations as they grow and acquire infiltrative proprieties [48]. In contrast, no association was found with any assessed variables in high-grade tumours. This may be explained by the different molecular pathways involved in high-grade tumours and their aggressive behaviour from the beginning [48]. Reinforcing the importance of tumour grade in neoplastic LN spread, tumour size did not correlate with CK19 mRNA positivity when stratified by grade or histological type.

The biologic significance of LN molecular tumour detection is of leading importance. Although the sole presence of small amounts of tumour cells in regional LN does not necessarily imply poor prognosis, it may indicate a yet undefined risk of disease recurrence. This would explain why, with 


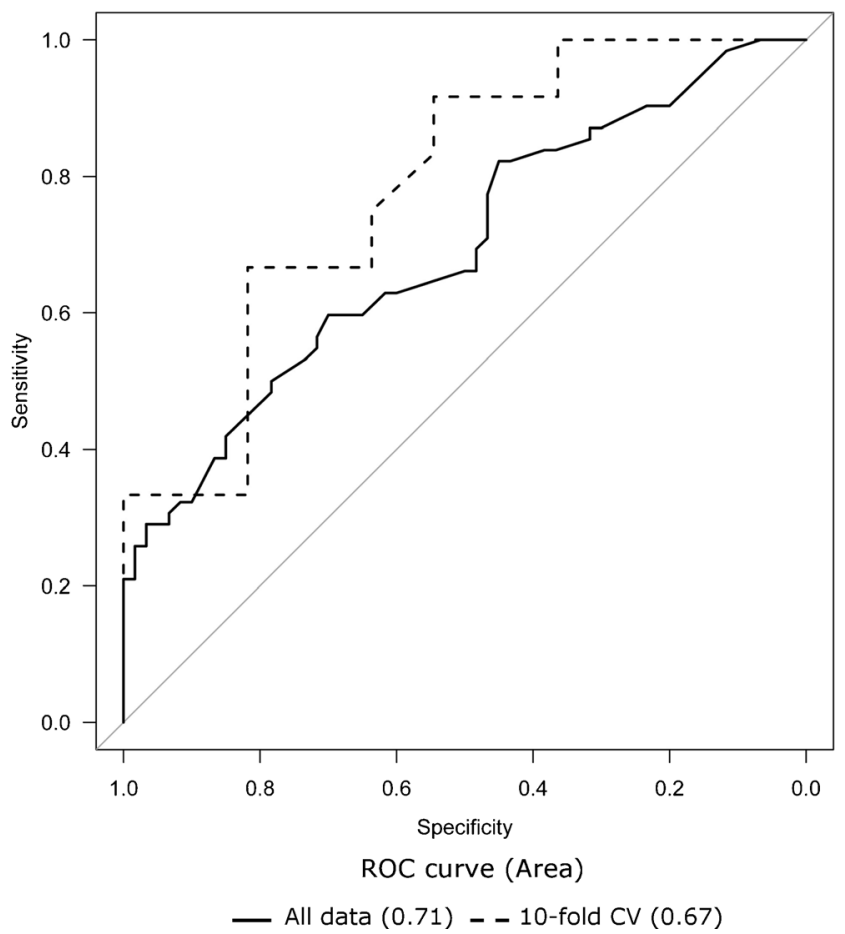

Fig. 4 ROC curve of the multivariate logistic regression model for OSNA results including gender, grade and the number of fresh lymph nodes as variables predicting molecular positivity. Continuous line: All data (0.71 (95\% CI = 0.62-0.79); Dashed line: 10 -fold CV (AUC $=0.67$ $[95 \% \mathrm{CI}=0.59-0.76])$ historical recurrence records of $2.5 \%$ in pT1 N0 tumours, we have found that almost $60 \%$ had some CK19 mRNA tumour burden in their LN $[11,49]$. In this context, low TTLs may have no further biologic consequences, but large amounts of tumour burden within the LN may imply higher odds of recurrence [7, 27]. A study using RT-qPCR detection of Guanylyl cyclase C (GUCY2C) by RT-qPCR found $87.5 \%$ patients with positive LN, although only $20.9 \%$ developed recurrent disease [7].

Quantifying the amount of tumour burden, not just the presence or absence of tumour, in regional lymph nodes is therefore important in prognosticating from molecular results, as has been stated in CRC [3, 7, 11, 20, 35] and breast cancer $[27,31]$. It should be highlighted that whereas the median TTL of our cohort was 2015 CK19 mRNA copies/ $\mu \mathrm{L}$, values up to $15,000 \mathrm{CK} 19 \mathrm{mRNA}$ copies/ $\mu \mathrm{L}$ have been recently set in breast cancer sentinel LN as clinically relevant to predict additional axillary LN metastases [27]. Some authors have also demonstrated the relationship between the amount of metastatic tumour and prognosis, stressing the importance of distinguishing ITC from micrometastases [7, 11, 23, 24, 50]. Two novel Japanese multicentre studies stress the need to stratify molecular outcomes in LN assessment. The first one addresses that TTL significantly increases with pN stage [28] and suggests it as a potential staging technique. The second one has shown the correlation of LN micrometastasis volume, measured by qRT-PCR for CEA mRNA, as a predictor of
Table 3 Total tumour load distribution in high and low-grade tumours

\begin{tabular}{|c|c|c|c|c|c|c|}
\hline \multirow[t]{2}{*}{ pT } & \multicolumn{4}{|c|}{ CK19 mRNA copies/ $\mu \mathrm{L}$ per case $n(\%)$} & \multirow[t]{2}{*}{ Total $n(\%)$} & \multirow[t]{2}{*}{$p$ value } \\
\hline & $<1000$ & $<2000$ & $<6000$ & $\geq 6000$ & & \\
\hline All cases & & & & & & 0.246 \\
\hline pT1 & $8(34.8)^{\mathrm{a}}$ & $6(26.1)$ & $6(26.1)$ & $3(13.0)$ & $23(100)$ & \\
\hline pT2 & $1(9.1)$ & $5(45.4)$ & $3(27.3)$ & $2(18.2)$ & $11(100)$ & \\
\hline pT3 & $9(24.3)$ & $6(16.2)$ & $8(21.6)^{\mathrm{a}}$ & $14(37.8)$ & $37(100)$ & \\
\hline pT4a & $2(40.0)$ & $1(20.0)$ & $2(40.0)$ & $0(0.0)$ & $5(100)$ & \\
\hline Total & $20(26.3)$ & $18(23.7)$ & $19(25)$ & $19(25)$ & $76(100)$ & \\
\hline High grade & & & & & & 0.61 \\
\hline pT1 & $1(20.0)$ & $1(20.0)$ & $2(40.0)$ & $1(20.0)$ & $5(100)$ & \\
\hline pT2 & $1(50.0)$ & $0(0.0)$ & $0(0.0)$ & $1(50.0)$ & $2(100)$ & \\
\hline pT3 & $1(12.5)$ & $4(50.0)$ & $1(12.5)$ & $2(25.0)$ & $8(100)$ & \\
\hline pT4a & $1(33.3)$ & $0(0.0)$ & $2(66.7)$ & $0(0.0)$ & $3(100)$ & \\
\hline Total & $4(22.2)$ & $5(27.8)$ & $5(27.8)$ & $4(22.2)$ & $18(100)$ & \\
\hline Low grade & & & & & & 0.01 \\
\hline pT1 & $6(35.3)$ & $5(29.4)$ & $4(23.5)$ & $2(11.8)$ & 17 (100) & \\
\hline pT2 & $0(0.0)$ & $5(55.6)$ & $3(33.3)$ & $1(11.1)$ & $9(100)$ & \\
\hline pT3 & $8(28.6)$ & $2(7.1)$ & $6(21.4)$ & $12(42.9)$ & $28(100)$ & \\
\hline pT4a & $1(50.0)$ & $1(50.0)$ & $0(0.0)$ & $0(0.0)$ & $2(100)$ & \\
\hline Total & $15(26.8)$ & $13(23.2)$ & $13(23.2)$ & $15(26.8)$ & $56(100)$ & \\
\hline
\end{tabular}

${ }^{\mathrm{a}}$ In two patients, tumour grade could not be assessed 
recurrence in stage II patients [51]. In addition to these findings, we have found that TTL unveils distinct progression patterns in low- and high-grade neoplasms.

Our study has some drawbacks; firstly, a follow-up period of 2 years is a limited time to establish the amount of CK19 mRNA copies that would have clinical significance; thus, the OSNA results could not be correlated with prognosis. Nevertheless, this study was not designed to determine the role of the molecular results as a prognostic factor, but to find out whether the OSNA results correlated with other classical CRC high-risk factors. Secondly, in order to evaluate the histological high-risk factors, we focused on histologically $\mathrm{pN} 0$ specimens. To assess the potential predictive value of the TTL, colectomy specimens should be studied regardless of their LN status, thus comparing histological and molecular evaluation methods and their ability to predict disease recurrence.

In conclusion, this study identifies the presence of undetected tumour burden in LN of early colon cancer patients. TTL correlates with other CRC classical high-risk factors and may be placed among them. We thereby support CK19 mRNA TTL as a fast and reliable approach to help better stage early colon cancer. Long-term follow-up and validation studies are needed to obtain a predictive prognostic scale for stage I-II colon cancer patients based on the patient's TTL.

$\mathrm{AC}$, adenocarcinoma; AUC, area under the curve; CK19, cytokeratin 19; CRC, colorectal cancer; FFPE, formalinfixation paraffin-embedding; HE, Haematoxylin and Eosin; IHC, immunohistochemistry; IQR, interquartile range; ITC, isolated tumour cells; LN, lymph node; OSNA, one-step nucleic acid amplification; $\mathrm{pT}$, tumour stage; ROC, receiveroperator characteristic; RT-LAMP, reverse transcription loopmediated isothermal amplification; TTL, total tumour load

\begin{abstract}
Acknowledgments We thank Mireia Aragay for her help with the language editing, Jordi Camps and Dr. Peter C. Burger for their help in revising the manuscript; Carla Montironi for contribution to collection of data and to Eva Fernandez, Mireia Garcia, Olga Luna and Judit Vela for their technical support.
\end{abstract}

Compliance with ethical standards The study protocol was approved by the Ethics and Scientific committee of each participating institution. All patients signed a written informed consent document for participation in the study. The study was performed in compliance with The Code of Ethics of the World Medical Association (Declaration of Helsinki).

Funding Work supported by the Banc de Tumors-Biobanc Hospital Clinic-IDIBAPS and Xarxa de Bancs de Tumors de Catalunya (XBTC), and by grants from the Fundación Científica de la Asociación Española Contra el Cáncer (GCB13131592CAST), Ministerio de Economía y Competitividad (SAF2014-54,453-R), Agència de Gestió d'Ajuts Universitaris i de Recerca (2014SGR135), and by Sysmex Coorp Spain (Sant Just Desvern, Spain). CIBERehd is funded by the Instituto de Salud Carlos III.

Conflict of interest The authors declare that they have no competing interests.
Open Access This article is distributed under the terms of the Creative Commons Attribution 4.0 International License (http:// creativecommons.org/licenses/by/4.0/), which permits unrestricted use, distribution, and reproduction in any medium, provided you give appropriate credit to the original author(s) and the source, provide a link to the Creative Commons license, and indicate if changes were made.

\section{References}

1. Benson AB, Venook AP, Bekaii-Saab T, et al. (2014) Colon cancer, version 3.2014. J Natl Compr Cancer Netw 12:1028-1059

2. Van Cutsem E, Oliveira J (2009) Primary colon cancer: ESMO clinical recommendations for diagnosis, adjuvant treatment and follow-up. Ann Oncol 20(Suppl 4):49-50. doi:10.1093 /annonc/mdp126

3. Rahbari NN, Bork U, Motschall E, et al. (2012) Molecular detection of tumor cells in regional lymph nodes is associated with disease recurrence and poor survival in node-negative colorectal cancer: a systematic review and meta-analysis. J Clin Oncol 30:60-70. doi:10.1200/JCO.2011.36.9504

4. Compton CC (2007) Optimal pathologic staging: defining stage II disease. Clin Cancer Res 13:6862s-70s. doi:10.1158/1078-0432. CCR-07-1398

5. Weitz J, Koch M, Debus J, et al. (2005) Colorectal cancer. Lancet 365:153-165. doi:10.1016/S0140-6736(05)17706-X

6. Weitz J, Kienle P, Magener A, et al. (1999) Detection of disseminated colorectal cancer cells in lymph nodes, blood and bone marrow. Clin Cancer Res 5:1830-1836

7. Hyslop T, Waldman SA (2013) Molecular staging of node negative patients with colorectal cancer. J Cancer 4:193-199. doi:10.7150 /jca. 5830

8. Visser M, Jiwa M, Horstman A, et al. (2008) Intra-operative rapid diagnostic method based on CK19 mRNA expression for the detection of lymph node metastases in breast cancer. Int J Cancer 122: 2562-2567. doi:10.1002/ijc.23451

9. Yamamoto H, Sekimoto M, Oya M, et al. (2011) OSNA-based novel molecular testing for lymph node metastases in colorectal cancer patients: results from a multicenter clinical performance study in Japan. Ann Surg Oncol 18:1891-1898. doi:10.1245 /s10434-010-1539-5

10. Iddings D, Ahmad A, Elashoff D, Bilchik A (2006) The prognostic effect of micrometastases in previously staged lymph node negative (N0) colorectal carcinoma: a meta-analysis. Ann Surg Oncol 13: 1386-1392. doi:10.1245/s10434-006-9120-y

11. Sloothaak DAM, Sahami S, van der Zaag-Loonen HJ, et al. (2014) The prognostic value of micrometastases and isolated tumour cells in histologically negative lymph nodes of patients with colorectal cancer: a systematic review and meta-analysis. Eur J Surg Oncol 40: 263-269. doi:10.1016/j.ejso.2013.12.002

12. Merkel S, Wein A, Günther K, et al. (2001) High-risk groups of patients with stage II colon carcinoma. Cancer 92:1435-1443

13. Sasaki M, Watanabe H, Jass JR, et al. (1997) Occult lymph node metastases detected by cytokeratin immunohistochemistry predict recurrence in "node-negative" colorectal cancer. J Gastroenterol 32: 758-764

14. Davies M, Arumugam PJ, Shah VI, et al. (2008) The clinical significance of lymph node micrometastasis in stage I and stage II colorectal cancer. Clin Transl Oncol 10:175-179

15. Nicastri DG, Doucette JT, Godfrey TE, Hughes SJ (2007) Is occult lymph node disease in colorectal cancer patients clinically significant? A review of the relevant literature. J Mol Diagn 9:563-571. doi:10.2353/jmoldx.2007.070032

16. Law CHL, Wright FC, Rapanos T, et al. (2003) Impact of lymph node retrieval and pathological ultra-staging on the prognosis of 
stage II colon cancer. J Surg Oncol 84:120-126. doi:10.1002 /jso.10309

17. Lips DJ, Koebrugge B, Liefers GJ, et al. (2011) The influence of micrometastases on prognosis and survival in stage I-II colon cancer patients: the Enroute $\oplus$ Study. BMC Surg 11:11. doi:10.1186 /1471-2482-11-11

18. Rosenberg R, Hoos A, Mueller J, et al. (2002) Prognostic significance of cytokeratin-20 reverse transcriptase polymerase chain reaction in lymph nodes of node-negative colorectal cancer patients. J Clin Oncol 20:1049-1055

19. Bilchik AJ, Hoon DSB, Saha S, et al. (2007) Prognostic impact of micrometastases in colon cancer: interim results of a prospective multicenter trial. Ann Surg 246:568-575 . doi:10.1097/SLA.0b013 e318155a9c7discussion 575-7

20. Sirop S, Kanaan M, Korant A, et al. (2011) Detection and prognostic impact of micrometastasis in colorectal cancer. J Surg Oncol 103:534-537. doi:10.1002/jso.21793

21. Tsujimoto M, Nakabayashi K, Yoshidome K, et al. (2007) One-step nucleic acid amplification for intraoperative detection of lymph node metastasis in breast cancer patients. Clin Cancer Res 13: 4807-4816. doi:10.1158/1078-0432.CCR-06-2512

22. Yamamoto N, Daito M, Hiyama K, et al. (2013) An optimal mRNA marker for OSNA (one-step nucleic acid amplification) based lymph node metastasis detection in colorectal cancer patients. Jpn J Clin Oncol 43:264-270. doi:10.1093/jjco/hys227

23. Croner RS, Geppert C-I, Bader FG, et al. (2014) Molecular staging of lymph node-negative colon carcinomas by one-step nucleic acid amplification (OSNA) results in upstaging of a quarter of patients in a prospective, European, multicentre study. Br J Cancer 110:2544 2550. doi:10.1038/bjc.2014.170

24. Vogelaar FJ, Reimers MS, van der Linden RLA, et al. (2014) The diagnostic value of one-step nucleic acid amplification (OSNA) for sentinel lymph nodes in colon cancer patients. Ann Surg Oncol 21: 3924-3930. doi:10.1245/s10434-014-3820-5

25. Güller U, Zettl A, Worni M, et al. (2012) Molecular investigation of lymph nodes in colon cancer patients using one-step nucleic acid amplification (OSNA): a new road to better staging? Cancer 118: 6039-6045. doi:10.1002/cncr.27667

26. Tamaki Y, Akiyama F, Iwase T, et al. (2009) Molecular detection of lymph node metastases in breast cancer patients: results of a multicenter trial using the one-step nucleic acid amplification assay. Clin Cancer Res 15:2879-2884. doi:10.1158/1078-0432.CCR-08-1881

27. Peg V, Espinosa-Bravo M, Vieites B, et al. (2013) Intraoperative molecular analysis of total tumor load in sentinel lymph node: a new predictor of axillary status in early breast cancer patients. Breast Cancer Res Treat 139:87-93. doi:10.1007/s10549-0132524-Z

28. Yamamoto H, Tomita N, Inomata M, et al. (2015) OSNA-assisted molecular staging in colorectal cancer: a prospective multicenter trial in Japan. Ann Surg Oncol:1-6. doi:10.1245/s10434-0154880-x

29. Cserni G (2012) Intraoperative analysis of sentinel lymph nodes in breast cancer by one-step nucleic acid amplification. J Clin Pathol 65:193-199. doi:10.1136/jclinpath-2011-200301

30. Rubio IT, Espinosa-Bravo M, Rodrigo M, et al. (2014) Nomogram including the total tumoral load in the sentinel nodes assessed by one-step nucleic acid amplification as a new factor for predicting nonsentinel lymph node metastasis in breast cancer patients. Breast Cancer Res Treat 147:371-380. doi:10.1007/s10549-014-3108-2

31. Espinosa-Bravo M, Sansano I, Pérez-Hoyos S, et al. (2013) Prediction of non-sentinel lymph node metastasis in early breast cancer by assessing total tumoral load in the sentinel lymph node by molecular assay. Eur J Surg Oncol 39:766-773. doi:10.1016/j. ejso.2013.03.011
32. Sobin LH, Gospodarowicz MK, Wittekind C (2009) UICC: TNM classification of malignant tumours, 7th edn. Wiley-Blackwell, New York

33. Washington MK, Berlin J, Branton P, et al. (2009) Protocol for the examination of specimens from patients with primary carcinoma of the colon and rectum. Arch Pathol Lab Med 133:1539-1551. doi:10.1043/1543-2165-133.10.1539

34. R Core Team (2014) R: A language and environment for statistical computing. Found. Stat. Comput. Vienna, Austria

35. Wanebo HJ, LeGolvan M, Paty PB, et al. (2012) Meeting the biologic challenge of colorectal metastases. Clin Exp Metastasis 29: 821-839. doi:10.1007/s10585-012-9517-x

36. O'Connor ES, Greenblatt DY, LoConte NK, et al. (2011) Adjuvant chemotherapy for stage II colon cancer with poor prognostic features. J Clin Oncol 29:3381-3388. doi:10.1200/JCO.2010.34.3426

37. Quah H-M, Chou JF, Gonen M, et al. (2008) Identification of patients with high-risk stage II colon cancer for adjuvant therapy. Dis Colon Rectum 51:503-507. doi:10.1007/s10350-008-9246-Z

38. Klein CA (2009) Parallel progression of primary tumours and metastases. Nat Rev Cancer 9:302-312. doi:10.1038/nrc2627

39. Compton CC, Fielding LP, Burgart LJ, et al. (2000) Prognostic factors in colorectal cancer. College of American Pathologists Consensus Statement 1999. Arch Pathol Lab Med 124:979-994. doi:10.1043/0003-9985(2000)124<0979:PFICC >2.0.CO;2

40. Goldstein NS (2002) Lymph node recoveries from 2427 pT3 colorectal resection specimens spanning 45 years: recommendations for a minimum number of recovered lymph nodes based on predictive probabilities. Am J Surg Pathol 26:179-189

41. Chang GJ, Rodriguez-Bigas MA, Skibber JM, Moyer VA (2007) Lymph node evaluation and survival after curative resection of colon cancer: systematic review. J Natl Cancer Inst 99:433-441. doi:10.1093/jnci/djk092

42. Swanson RS, Compton CC, Stewart AK, Bland KI (2003) The prognosis of T3N0 colon cancer is dependent on the number of lymph nodes examined. Ann Surg Oncol 10:65-71

43. Dhar DK, Yoshimura H, Kinukawa N, et al. (2005) Metastatic lymph node size and colorectal cancer prognosis. J Am Coll Surg 200:20-28. doi:10.1016/j.jamcollsurg.2004.09.037

44. Märkl B, Rößle J, Arnholdt HM, et al. (2012) The clinical significance of lymph node size in colon cancer. Mod Pathol 25:14131422. doi:10.1038/modpathol.2012.92

45. Hyslop T, Weinberg DS, Schulz S, et al. (2012) Analytic lymph node number establishes staging accuracy by occult tumor burden in colorectal cancer. J Surg Oncol 106:24-30. doi:10.1002 /jso.23051

46. Soni M, Wiese D, Korant A, et al. (2011) Comparison of nodal positivity between SLNM vs conventional surgery in colon cancer patients with $<12$ and $\geq 12$ lymph nodes harvested. Am J Surg 202: 207-213. doi:10.1016/j.amjsurg.2010.06.028

47. Cahill RA, Lindsey I, Cunningham C (2010) Sentinel node mapping by colonic tattooing. Surg Endosc 24:2365-2366. doi:10.1007 /s00464-010-0941-1

48. Hanahan D, Weinberg RA (2011) Hallmarks of cancer: the next generation. Cell 144:646-674. doi:10.1016/j.cell.2011.02.013

49. Gunderson LL, Jessup JM, Sargent DJ, et al. (2010) Revised TN categorization for colon cancer based on national survival outcomes data. J Clin Oncol 28:264-271. doi:10.1200/JCO.2009.24.0952

50. Jeffers MD, O’Dowd GM, Mulcahy H, et al. (1994) The prognostic significance of immunohistochemically detected lymph node micrometastases in colorectal carcinoma. J Pathol 172:183-187. doi:10.1002/path.1711720205

51. Yamamoto H, Murata K, Fukunaga M, et al. (2016) Micrometastasis volume in lymph nodes determines disease recurrence rate of stage II colorectal cancer: a prospective multicenter trial. Clin Cancer Res. doi:10.1158/1078-0432.CCR-15-2199 